Uta Quasthoff, Vivien Heller, Miriam Morek

\title{
Diskurskompetenz und diskursive Partizipation als Schlüssel zur Teilhabe an Bildungsprozessen
}

\section{Grundlegende Konzepte und Untersuchungslinien}

\begin{abstract}
Dieses Kapitel stellt den theoretischen, in der Interaktionalen Diskursanalyse verorteten Rahmen des Bandes dar: Wir gehen von der Annahme aus, dass sich gesellschaftliche und bildungsbezogene Teilhabe im Kleinen durch die Art der Partizipation an wiederkehrenden Interaktionssituationen, genauer: an diskursiven, gattungsförmig verfestigten Praktiken realisiert. Dabei können unterschiedliche Passungsverhältnisse zwischen schulischen und außerschulischen diskursiven Praktiken für den Einzelnen förderlich oder hinderlich sein. Mit dem Fokus auf Mündlichkeit stellen wir den Zusammenhang von Partizipationsstrukturen und -gelegenheiten dar, die externe Ressourcen für den Erwerb von Diskurskompetenzen bilden. Wir zeigen anschließend, dass Kindern und Jugendlichen entsprechende Ressourcen in sehr unterschiedlicher Weise zur Verfügung stehen und erläutern, was dies für diskursive Partizipations- und Erwerbsgelegenheiten im Unterricht bedeutet. Daraus ergibt sich abschließend die Frage, wie sich die beschriebenen Forschungsbefunde der Diskurserwerbs- und linguistischen Unterrichtsforschung in Maßnahmen zur Förderung der diskursiven Partizipation insbesondere bildungsbenachteiligter Kinder und Jugendlicher umsetzen lassen.
\end{abstract}

Keywords: Partizipation, diskursive Praktiken, Gattungen, Diskurskompetenz, Heterogenität, Passung

\section{Einleitung}

Nach wie vor prägen soziale und zuwanderungsbedingte Unterschiede den Bildungserfolg von Kindern und Jugendlichen in Deutschland. Insbesondere

\footnotetext{
Uta Quasthoff, TU Dortmund, Emil-Figge-Straße 50, 44227 Dortmund, uta.quasthoff@tu-dortmund.de

Vivien Heller, Bergische Universität Wuppertal, Gaußstraße 20, 42119 Wuppertal, vheller@uni-wuppertal.de

Miriam Morek, Universität Duisburg-Essen, Universitätsstr. 12, 45141 Essen, miriam.morek@uni-due.de
}

Ә Open Access. ( 2021 Uta Quasthoff, et al., publiziert von De Gruyter. (cc) BY-NC-ND Dieses Werk ist lizensiert unter einer Creative Commons Namensnennung - Nicht-kommerziell - Keine Bearbeitung 4.0 International Lizenz. 
für die sprachlichen Domänen sind Korrelationen zwischen einem höheren Sozialstatus und höheren Kompetenzwerten empirisch belegt (Stanat et al. 2016, 2017). Verantwortlich für diesen Zusammenhang sind vor allem die unterschiedlichen häuslichen Bedingungen für die Aneignung der für Bildungsteilhabe erforderlichen sprachlichen Ressourcen gerade auch im Bereich des Mündlichen (Quasthoff, Wild et al. in diesem Band). Der Schule gelingt es bislang also oft nur unzureichend, den unterschiedlichen milieubedingten Voraussetzungen so zu begegnen, dass ihren Schülerinnen und Schülern eine chancengleiche Teilhabe an Bildung, Arbeit, politischen Prozessen und Kulturgütern eröffnet wird.

Schon seit den 1970er Jahren zeigen insbesondere die Kultur- und Bildungssoziologie (Bourdieu \& Passeron 1977; Bourdieu 1977; Lareau 2003; Kramer \& Helsper 2010), die Soziolinguistik (Bernstein 1977) sowie die ethnographisch orientierte Spracherwerbs- und Sozialisationsforschung (Heath 1983; Hoff-Ginsberg 1991; Hart \& Risley 1999; Blum-Kulka \& Snow 2002; Grøver et al. 2019; Burdelski \& Howard 2020), welche entscheidende Rolle Sprache und Sprechen für Benachteiligungen in der Teilhabe an schulisch vermittelten Bildungsprozessen spielen. Ein wesentlicher Mechanismus der Reproduktion von Bildungsungleichheit wurde und wird in den Passungen und Divergenzen zwischen herkunftsbedingten sprachlich-diskursiven Praktiken (vgl. zu diesem und anderen grundlegenden Konzepten das Glossar in diesem Band) und den schulischen Erwartungen und Anforderungen gesehen (Blum-Kulka \& Snow 2002; Heath 1983; Heller 2012; Morek 2012; Quasthoff \& Morek 2015).

Zentral für die Teilhabe an komplexeren Bildungs- und Lernprozessen sind aus neuerer Sicht insbesondere diskursive Praktiken wie Erzählen, Erklären und Argumentieren: Diese über Wortschatz und Grammatik hinausgehenden sprachlichen Verfahren stellen einerseits ein wesentliches Medium des Lernens in allen Fächern der weiterführenden Schulen dar (vgl. Heller in diesem Band; Morek \& Heller in diesem Band; Prediger in diesem Band). Andererseits sind die familialen Erwerbskontexte für diese komplexen sprachlichen Fähigkeiten ebenfalls nachweislich in verschiedenen Familienmilieus sehr unterschiedlich ausgeprägt (vgl. Abschnitt 5 unten; Morek in diesem Band). Dementsprechend weisen die außerschulisch erworbenen diskursiven Praktiken von Kindern und Jugendlichen unterschiedliche Grade der Passung zu unterrichtlichen Erwartungen auf. Der adaptive Umgang mit den unterschiedlichen Passungsverhältnissen und das Schaffen diskursiver Partizipationsgelegenheiten für alle Schülerinnen und Schüler dürfte demnach eine der entscheidenden Stellschrauben sein, um Mechanismen der Reproduktion von Bildungsungleichheit entgegenzuwirken. Wir konzentrieren uns deshalb in diesem Beitrag wie auch im Band auf diskursive Anforderungen und Ressourcen im Medium der Mündlichkeit und beleuchten schriftliche Kompetenzen als ihre Folge. 
Vor dem hier skizzierten Hintergrund sind in den letzten Jahren mehrere Studien entstanden, die mit Bezug auf deutschsprachige Diskurserwerbs- und Diskurssozialisationsprozesse untersuchen, wie Kinder und Jugendliche in schulischen und außerschulischen Kontexten an diskursiven Praktiken erfolgreich oder nur eingeschränkt partizipieren. Einige dieser Studien bilden den Hintergrund dieses Bandes. Das vorliegende Kapitel hat das Ziel, die thematische Klammer dieser Studien herauszuarbeiten und ihre gemeinsamen theoretischen Bezugspunkte zu explizieren. Die für den Band zentralen Begriffe sind zusätzlich in einem Glossar erläutert; grundlegende methodische Aspekte werden in einem eigenen Kapitel (Quasthoff in diesem Band) beschrieben.

Im Einzelnen legen wir in diesem Kapitel zunächst ein Verständnis von ,Partizipation“ dar (Abschnitt 2), das in der Interaktionalen Diskursanalyse (Quasthoff, Heller \& Morek 2017) verortet ist. Diese fußt auf der ethnomethodologischen Konversationsanalyse und fokussiert auf global organisierte diskursive Praktiken bzw. Gattungen in Gesprächen. In Abschnitt 3 beschreiben wir auf dieser Basis, inwiefern die für die jeweiligen Gattungen typischen Partizipationsstrukturen von erfahrenen Interaktionsbeteiligten genutzt werden können, um Partizipationsgelegenheiten für weniger kompetente Interaktionsbeteiligte $\mathrm{zu}$ schaffen. Hier wird die Verbindung zwischen Partizipationsstrukturen und Erwerbschancen grundgelegt. Abschnitt 4 widmet sich den sprachlich-diskursiven Anforderungen, die Interaktanten beim Vollzug diskursiver Praktiken zu bewerkstelligen haben und an deren Bewältigung sich ,Diskurskompetenz' bemisst. Wir beschreiben hier genauer die Mechanik des Erwerbs durch Partizipation. Ausgehend von diesen grundsätzlichen diskurserwerbstheoretischen Annahmen vor allem zu externen Ressourcen des Erwerbs skizzieren wir schließlich, dass Kindern und Jugendlichen entsprechende Ressourcen in sehr unterschiedlicher Weise zur Verfügung stehen, und erläutern, was dies für diskursive Partizipationsgelegenheiten im Unterricht bedeutet (Abschnitt 5). Daraus ergibt sich ein Ausblick auf die Frage, wie sich die beschriebenen Forschungsbefunde der Diskurserwerbs- und linguistischen Unterrichtsforschung in Maßnahmen umsetzen lassen, um die diskursive Partizipation insbesondere bildungsbenachteiligter Kinder und Jugendlicher zu erhöhen (Abschnitt 6).

\section{Partizipation an diskursiven Praktiken als Schlüssel zur Teilhabe}

Unser Verständnis von Partizipation speist sich aus mikrosoziologischen und ethnomethodologischen Ansätzen (Garfinkel 1967). Gesellschaftliche Teilhabe realisiert 
sich demnach im Kleinen durch die Art der täglichen Partizipation an Interaktionspraktiken. Wir reservieren den Begriff ,Teilhabe‘ dabei für den Zugang zu Bildungsund anderen gesellschaftlichen Angeboten und sprechen demgegenüber von ,Partizipation‘, wenn wir die Beteiligung des Einzelnen an konkreten Interaktionsprozessen meinen. Es geht dabei also um die Frage, auf welche Weise Gesellschaftsmitglieder (members) in die Aktivitäten, die ihre Lebenswelt (Schütz \& Luckmann 1975) prägen, interaktiv involviert sind, und wie diese Interaktionserfahrungen sie langfristig zur Teilhabe an gesellschaftlichen Prozessen befähigen.

Die meisten Aktivitäten der unmittelbaren und mittelbaren Lebenswelt werden sprachlich und im Rahmen diskursiver Praktiken vollzogen. Den Terminus ,diskursive Praktiken` benutzen wir als Oberbegriff für interaktiv vollzogene sprachliche Aktivitäten, die global organisiert sind (s. u.) und die oftmals den Status verfestigter kommunikativer Gattungen bzw. Genres haben, z. B. Berichten, Erzählen, Erklären und Argumentieren. Unter kommunikativen Gattungen werden in der Wissenssoziologie gesellschaftlich verfestigte Lösungsverfahren für wiederkehrende kommunikative Probleme verstanden (Luckmann 1986, 1989; Günthner \& Knoblauch 1996). So sind rekonstruktive Gattungen wie das Berichten und Erzählen insbesondere darauf zugeschnitten, das kommunikative Problem des Teilens und Einordnens von Begebenheiten und Erfahrungen zu lösen (Quasthoff 1980; Bergmann 1987; Günthner 2005); das Erklären ist ein Verfahren, mit dem Probleme des Wissenstransfers und der Wissensdemonstration bearbeitet werden können (Goldberg 1975; Psathas 1992; Keppler \& Luckmann 1991; Kotthoff 2009; Morek 2012); das Argumentieren schließlich ist darauf ausgerichtet, das Aushandeln divergenter Geltungsansprüche zu ermöglichen (Goodwin \& Goodwin 1987; Knoblauch 1991; Spranz-Fogasy 2003; Heller 2012; zusammenfassend: Morek, Heller \& Quasthoff 2017). Kompetente Mitglieder von Sprachgemeinschaften müssen also für die genannten Probleme nicht immer wieder von Neuem Lösungen entwickeln, sondern können mit Gattungen auf einen geteilten kommunikativen „Orientierungsrahmen“ (Günthner 1995: 196) zurückgreifen. Dazu müssen sie diesen jedoch kennen und kontextuell anpassen können.

Beim Erzählen, Erklären und Argumentieren handelt es sich nun insofern um vergleichsweise komplexe Praktiken, als diese auf Grund ihrer globalen Organisationsform eine mehrteilige sequenzielle Organisation aufweisen. In sequenzieller Hinsicht bilden sie Diskurseinheiten (Wald 1978) und werden innerhalb von Gesprächen ein- und ausleitend als Aktivität eines bestimmten Gattungstyps erkennbar gemacht. Sie verlangen entsprechend eine - jeweils gattungsspezifische gesprächsorganisatorische Vor- und Nachbereitung wie auch eine bestimmte Art des interaktiven Vollzugs.

Dabei orientieren sich die Beteiligten an der Bearbeitung gesprächsstruktureller Jobs (Psathas 1992; Hausendorf \& Quasthoff 1996; Sacks 1995: IIXX), die da- 
rauf zugeschnitten sind, das jeweilige kommunikative Problem zu lösen, für dessen Bearbeitung sich die Gattung entwickelt hat. Beim Erzählen und Erklären beinhaltet dies, dass im Zuge der gesprächsorganisatorischen Vorbereitung einem der Beteiligten temporär die Rolle des primären Sprechers (Wald 1978) zugewiesen wird. Dabei wird mittels eines globalen Zugzwangs (Hausendorf \& Quasthoff 1996: 115) das Folgen eines narrativen oder explanativen Äußerungspakets (Sacks 1995: 354-359) konditionell relevant gesetzt, d. h. kommunikativ erwartbar gemacht: Fragen wie „Was ist dir denn gestern im Zug passiert?“ bzw. „Warum soll man Schokolade eigentlich im Wasserbad schmelzen lassen?“ sind jeweils globale Zugzwänge, die in eine Erzählung bzw. Erklärung steuern. Während der Produktion eines solchen Äußerungspakets, d. h. des äußerungsübergreifenden Redezugs innerhalb der Durchführung von Erklär- oder Erzählaktivitäten, werden die gewöhnlichen, lokal operierenden Regeln der Rederechtsorganisation suspendiert, so dass die in der Rezipientenrolle Beteiligten lediglich Zuhöraktivitäten realisieren und ansonsten das Rederecht nicht für anderweitige Gesprächsbeiträge beanspruchen. Beim Argumentieren ist der Fall etwas anders gelagert: Hier haben die Beteiligten in den Rollen ,Proponent‘ und ,Opponent‘ mehr oder wenig gleichberechtigte Ansprüche auf das Rederecht, so dass argumentative Äußerungspakete, aber auch lokal operierende Widersprüche, Infragestellungen o. Ä. in beiden Rollen vorgebracht werden können. Für alle diskursiven Gattungen gilt gleichermaßen: Die Art und Weise, in der die gattungsspezifischen Jobs jeweils bearbeitet werden, ist dabei nicht immer gleich, sondern verlangt den Zuschnitt der genutzten Mittel und Formen auf die kommunikativen Anforderungen eines bestimmten sozialen und kommunikativen Kontextes.

Die sozialen Kontexte, die für Kinder und Jugendliche kulturell und sprachlich prägend sind, zeichnen sich also jeweils durch bestimmte diskursive Praktiken aus (vgl. Abschnitt 4). Familiale Tischgespräche beispielsweise leben vor allem von Klatsch (Bergmann 1987), Erzählen (Blum-Kulka 1997), Erklären (Morek 2012), aber auch Konflikten und Formen des Argumentierens (Pontecorvo \& Fasulo 1997; Heller 2012; Goodwin \& Cekaite 2018; vgl. Morek in diesem Band). Für Peer-Interaktionen sind auf szenische Performanz angelegte narrative Praktiken besonders einschlägig (Morek 2014, 2018, in diesem Band). Habitualisiert in bestimmten Kommunikationsgemeinschaften und sozialen Kontexten vorkommende Gattungen lassen sich als Gattungsrepertoires (Günthner 2009; Heller 2012, Morek in diesem Band) ermitteln. Als zentral für das diskursive Repertoire mündlicher Unterrichtskommunikation erweisen sich insbesondere die Gattungen des Erklärens und Argumentierens (vgl. Heller et al. 2017; Morek 2016), insofern deren kommunikative Zwecke mit der Konstruktion, Demonstration und Aushandlung allgemeingültigen Wissens und Geltens zu tun haben. 
Mit dieser Sicht auf diskursive Gattungen als konstitutive Bestimmungsstücke des Handelns u. a. in Bildungsinstitutionen schließen wir an ein bestimmtes Verständnis des inzwischen sehr verbreiteten Begriffs ,Bildungssprache‘ an. Unter diesem Terminus bzw. mit vergleichbaren Konzepten (z. B. academic language oder language of schooling, vgl. Feilke 2012; Gogolin 2010; Heller \& Morek 2015a; Schleppegrell 2012) wurde in der Folge des PISA-Schocks die Rolle von Sprache bei der Reproduktion herkunftsbedingter Bildungs- und Teilhabechancen genauer untersucht. Damit war zunächst vor allem ein Fokus auf Schriftsprache und auf sprachstrukturelle Aspekte (z. B. Passiv, komplexe Nominalphrasen, Komposita) verbunden. Der vorliegende Band greift hingegen eine Weiterentwicklung des Begriffs im Sinne ,bildungssprachlicher Praktiken` auf (Morek \& Heller 2012, 2019): Damit wird dem Umstand Rechnung getragen, dass auch in mündlicher Kommunikation bildungssprachlich agiert wird, und dass neben syntaktischen und lexikalischen Aspekten die Diskursebene besonders relevant ist.

Bildungssprachliche Praktiken umfassen nach unserem Verständnis somit ein Repertoire diskursiver Praktiken, die sich für Zwecke der Wissenskonstruktion, -absicherung und -vermittlung verfestigt haben und bestimmte interaktive und sprachliche Anforderungen (s. u.) beinhalten. Zugleich berücksichtigt der Begriff der bildungssprachlichen Praktiken auch, dass mit Aktivitäten wie z. B. Erklären und Argumentieren stets auch Prozesse der Darstellung und Zuschreibung bildungsaffiner Identitäten verbunden sind (vgl. Morek \& Heller 2012, 2019).

Für uns ist daher bei der genaueren Rekonstruktion des Zusammenhangs von bildungssprachlichen Praktiken und Bildungsungleichheit zentral, nicht nur die diskursiven Erfahrungen verschiedener Schülerinnen und Schüler im schulischen Bereich zu fokussieren, sondern diese im Hinblick auf Erwerbschancen mit außerschulischen Erfahrungen systematisch zu vergleichen (vgl. Domenech \& Krah in diesem Band; Morek in diesem Band; Quasthoff, Wild et al. in diesem Band). Leitend sind dabei die Fragen: An welchen (bildungssprachlich relevanten) Praktiken können Schülerinnen und Schüler in bestimmten Kontexten überhaupt diskursiv partizipieren? Und wie genau partizipieren sie?

\section{Partizipationsstrukturen und Partizipationsgelegenheiten}

Einer der ersten, der - allerdings noch nicht in Bezug auf Gattungen, sondern auf einzelne Äußerungen - mit dem Partizipationsbegriff arbeitete, war Goffman (1981). Er prägte für die unterschiedlichen Beteiligungsrollen von (ratifizierten 
und nicht ratifizierten) Rezipientinnen und Rezipienten den Begriff participation framework und für die der Produzentinnen und Produzenten (Animator, Autor und Urheber einer Äußerung) den des production format. Beide Aspekte wurden später von Levinson (1988) unter dem Begriff participation zusammengeführt. Die Untersuchung von Partizipationsstrukturen bezieht sich in diesem Verständnis auf die Frage, in welchen interaktiven Beteiligungsrollen, entlang welcher Interaktionsmuster (vgl. Abschnitt 4) und mit welcher Art von Beiträgen Interaktantinnen und Interaktanten in diskursive Praktiken involviert sind. Dieses Verständnis impliziert, wie Goodwin \& Goodwin (2004) zeigen, dass derartige Partizipations- oder Beteiligungsrahmen dynamisch sind, d. h. sequenziell - Zug um Zug - hervorgebracht und an die Zwecke der konkreten Aktivität angepasst werden. Partizipation ist demnach zu charakterisieren als "temporally unfolding process through which separate parties demonstrate to each other their ongoing understanding of the events they are engaged in by building actions that contribute to the further progression of the very same events" (Goodwin 2007: 24-25).

Partizipation an diskursiven, global organisierten Praktiken ist nun zum Teil vorstrukturiert durch die für die jeweilige Praktik typische sequenzielle Organisation. $\mathrm{Zu}$ diesen Partizipationsstrukturen zählen zuallererst interaktive Rollen, die für die jeweilige Praktik konstitutiv sind. So nimmt etwa beim Erklären eine/r der Beteiligten als primäre/r Sprecher/in die interaktive Rolle des/ der Erklärenden ein, während andere als Erklärrezipienten beteiligt sind. Letztere können sich gemäß ihrer Rolle jeweils nur mit bestimmten Arten von Beiträgen - Nachfragen, verbalen oder nonverbalen Verstehensbekundungen (Quasthoff 1981; Morek 2012) - einbringen.

Insofern als im vorliegenden Band die Teilhabe von Kindern und Jugendlichen an Erwerbs- und Bildungsprozessen thematisch im Mittelpunkt steht, sind Partizipationsstrukturen allerdings noch aus einer anderen Perspektive relevant, und zwar als Partizipationsgelegenheiten. Inwieweit jemand an komplexen diskursiven Praktiken wie Erklären oder Argumentieren zu partizipieren vermag, ist einerseits abhängig von den jeweils verfügbaren sprachlichdiskursiven Ressourcen, die zur Partizipation benötigt werden, und andererseits von den Beteiligungsmöglichkeiten, die dem Kind kontextuell eröffnet werden. Da Partizipation i. d. S. aus der Sicht interaktiver Erwerbstheorien die Bedingung für den Erwerb entsprechender sprachlich-diskursiver Kompetenzen ist, kommt den Involvierungs- und Unterstützungsaktivitäten erwachsener Gesprächspartnerinnen und Gesprächspartner eine besondere Rolle zu. Die Frage, inwiefern etwa Eltern oder Lehrpersonen im Rahmen diskursiver Praktiken Partizipationsgelegenheiten für noch nicht vollständig kompetente members (Quasthoff 2011) eröffnen, ist also bezogen auf das einzelne Kind zugleich die Frage nach außerschulischen und unterrichtlichen Erwerbschancen. 
Aus der Diskurserwerbsforschung wissen wir, dass dabei nicht nur entscheidend ist, dass das Kind überhaupt in die Aktivitäten involviert wird, sondern vor allem, wie dies geschieht (Hausendorf \& Quasthoff 1996): Partizipationsgelegenheiten sind vor allem dann erwerbsförderlich, wenn das Kind in seiner diskursiven Partizipation zugleich gefordert und interaktiv unterstützt wird (Kern \& Quasthoff 2007) (vgl. Abschnitt 4).

Haben zunächst Familieninteraktionen allein das Potenzial, erwerbsförderliche Partizipationsgelegenheiten zu schaffen, so treten im Verlauf der Entwicklung Interaktionen in Kindergarten, Cliquen und Schule als Erwerbskontexte hinzu, die bisher unterschiedlich intensiv erforscht wurden. Gerade die Rolle des Unterrichts auch als informeller Spracherwerbskontext ist noch nicht lange erkannt (vgl. aber Harren 2015; Heller \& Morek 2015b; Kleinschmidt-Schinke 2018). Das liegt vor allem daran, dass große Teile der Unterrichtsforschung in den letzten Jahren auf Qualitätsaspekte von Unterricht fokussiert haben (z. B. kognitive Aktivierung, Klassenführung, Klassenklima), dabei jedoch die Sprachlichkeit von Unterrichtsgesprächen weniger stark in den Blick genommen haben (vgl. aber z. B. Pauli \& Reusser 2015). Hierin liegt demgegenüber die besondere Stärke eines linguistisch fundierten Zugangs zu Unterrichtskommunikation: Er kann das Zusammenspiel sprachlicher und fachlicher Anforderungen im Unterricht erhellen und zeigen, inwiefern Interaktion den Erwerb diskursiver Kompetenzen im Unterricht unterstützen kann.

Während diskursive Praktiken wie Erklären und Argumentieren im Unterricht in ihrer Rolle als Lernmedium von den Beteiligten oftmals unhinterfragt bleiben, wird aus linguistischer Sicht deutlich: Insbesondere das Erklären und Argumentieren sind für die Konstruktion, Vermittlung und Überprüfung fachlichen Wissens in Unterrichtsgesprächen absolut zentral. Die dafür erforderlichen explanativen und argumentativen Diskurskompetenzen werden allerdings von Lehrkräften meist umstandslos vorausgesetzt (Heller \& Quasthoff in diesem Band). Die Konsequenz ist, dass die unterrichtlichen Partizipationsgelegenheiten, die Voraussetzung für den Erwerb und Ausbau von bildungssprachlichen Diskursfähigkeiten sind, im besten Fall zufällig entstehen. Hinzu kommt, dass sie oftmals nur Schülerinnen und Schülern zur Verfügung gestellt werden, deren Beiträge inhaltlich ,passen', die also bereits über ein hinreichendes Maß an Diskurskompetenz verfügen, um am Klassengespräch zu partizipieren (Leßmann in diesem Band). Klette et al. (2018) entwickeln den Begriff der participation economy, um die ungleichen, auf wenige Schülerinnen und Schüler beschränkten Partizipationsgelegenheiten an fachlich und diskursiv anspruchsvollen Aktivitäten analytisch zu beschreiben. 
Insgesamt kommt Partizipationsgelegenheiten somit eine Doppelfunktion zu: Sie sind Voraussetzung und Werkzeug des Erwerbs diskursiver Kompetenzen. Wie wir den Zusammenhang von Bildungsteilhabe, diskursiver Passung und interaktiver Partizipation grundsätzlich konzipieren, ist in Abb. 1 schematisch dargestellt.

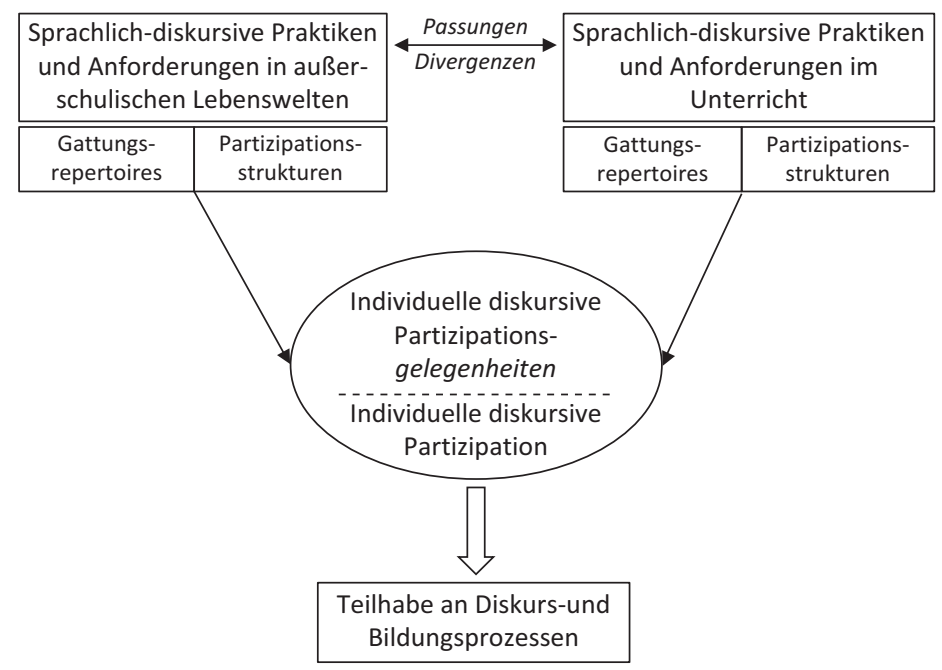

Abb. 1: Diskursive Partizipation als Schlüssel zur Teilhabe.

Die Teilhabe an unterrichtsvermittelten Bildungsprozessen wird jedoch aus unserer Sicht nicht nur von den individuellen Gelegenheiten zur erwerbsförderlichen Partizipation in den Beteiligungsrollen von Erklärenden und Argumentierenden bestimmt. Ob Gelegenheiten von Schülerinnen und Schülern genutzt und von Lehrkräften überhaupt - für alle oder nur für ausgewählte Lernende - geschaffen werden, hängt von weiteren Mechanismen ab. Dazu zählen implizite Orientierungen mit Blick auf die soziale Ordnung des Klassenverbandes und das Selbstverständnis, aus dem heraus das institutionelle Unterrichtsgeschehen insgesamt aus der Sicht der institutionellen Rollen Lehrkraft bzw. Schüler/in interpretiert und perspektiviert wird. Wir knüpfen an Ansätze der Bildungs- und Kultursoziologie (s. o.) an, wenn wir untersuchen, was Lehrende und Lernende in der Betrachtung von unterrichtlichem Handeln jeweils relevant setzen und wie sie die Handelnden implizit sozial kategorisieren (vgl. Heller \& Quasthoff in diesem Band). Daraus ergeben sich Konsequenzen dafür, wie Lehrkräfte mangelnde Passungen zwischen ihren Erwartungen und den Partizipationsfähigkeiten und -haltungen 
verschiedener Kinder erkennen und bearbeiten können (vgl. Morek \& Heller in diesem Band). Mit diesem Zugriff wird unser Wissen über Passungsverhältnisse also empirisch und explanativ erweitert.

\section{Diskurskompetenz und Ressourcen ihres Erwerbs}

Voraussetzung wie auch Ergebnis diskursiver Partizipation ist Diskurskompetenz. Unter Diskurskompetenz verstehen wir die Fähigkeit, produktiv und rezeptiv an global organisierten, d. h. diskursiven Praktiken gemäß den gattungsspezifischen Anforderungen interaktiv und sprachlich kontextualisierend partizipieren zu können. Dazu gehören folgende drei Teilfähigkeiten: Beteiligte müssen zum einen in der Lage sein, sich wechselseitig anzuzeigen, welchen Typ von diskursiver Praktik (d. h. welche Gattung) sie miteinander vollziehen. Dies beinhaltet wesentlich den Umgang mit globalen Zugzwängen: Beteiligte müssen u. a. erkennen, wann die Produktion eines ,größeren“ Äußerungspakets eines bestimmten Typs konditionell relevant gesetzt ist und wie es global anzuschließen ist. Die Beherrschung der Mechanik globaler sequenzieller Erwartungen kann sich auch darin zeigen, sequenzielle Kontexte bzw. Anschlussstellen für Äußerungspakete selbst zu schaffen. Insgesamt bezeichnen wir die hier beschriebene diskursive Teilfähigkeit als Kontextualisierungskompetenz.

Zum anderen verlangt die Partizipation an diskursiven Praktiken, dass die Beteiligten aus ihrer jeweiligen interaktiven Rolle heraus (z. B. Erklärproduzent/in oder -rezipient/in) zu den gesprächsstrukturellen Jobs beitragen. Dazu gehört auch kommunikatives Wissen über die unterschiedlichen Vertextungsmuster und -verfahren, mittels derer gattungsspezifische Äußerungspakete intern strukturiert werden (vgl. Heller in diesem Band). So werden Erzählungen meist chronologisch bzw. auf einen Planbruch zulaufend strukturiert, während Erklärungen und Argumentationen mehr oder weniger logische und hierarchisierende Strukturierungen (bspw. Formulieren einer Hypothese - Entwerfen eines Szenarios zur Veranschaulichung - Schlussfolgerung) zugrunde liegen. Den produktiven und rezeptiven Umgang mit derartigen gattungsorientierten Strukturierungsmustern bezeichnen wir als Vertextungskompetenz.

Die dritte Facette von Diskurskompetenz bildet die Markierungskompetenz. Sie beinhaltet die Fähigkeit, mittels sprachlicher, prosodischer und gestischer Formen die Gattung und die Struktur des jeweiligen Äußerungspakets für die Interaktionspartnerinnen und -partner erkennbar zu machen. Eine solche Markierung ist notwendig für die wechselseitige Koordination bei der gemeinsamen 
Bearbeitung der gesprächsstrukturellen Aufgaben (für eine ausführliche Darstellung der Kompetenzfacetten vgl. Quasthoff et al. 2019).

In welchem Ausmaß Kinder und Jugendliche bereits über diskursive Kompetenzen in den drei Teildimensionen und somit über interne Ressourcen zu diskursiver Partizipation verfügen, ist nicht leicht feststellbar: Schließlich ist an diskursiven Praktiken immer mindestens ein/e weitere/r Interaktant/in beteiligt, dessen/deren Gesprächsbeiträge sich mit denen des Kindes verweben und zum gemeinsamen Aufbau entsprechender Gesprächssequenzen beitragen (Quasthoff et al. 2019). Die Beiträge des vorliegenden Bandes greifen zur Rekonstruktion individueller Kompetenz auf der Basis interaktiv hergestellter Gesprächssequenzen auf den analytischen Zugang GLOBE zurück (vgl. auch Quasthoff in diesem Band; Quasthoff, Heller \& Morek 2017). Er ist kennzeichnend für das Vorgehen der Interaktionalen Diskursanalyse, insofern er die ethnomethodologische Beschreibung sequenzieller, interaktiver Strukturen in Gesprächen mit dem Blick auf die globale Ebene von Diskurspraktiken unter Erwerbsperspektive verbindet.

Unterschieden werden in GLOBE drei Ebenen der Beschreibung: (1) die der größeren, nacheinander zu bearbeitenden Interaktionsaufgaben (Jobs), die sich bei Diskurspraktiken allen Beteiligten stellen (z. B. Etablieren von Dissens und Etablieren von Begründungspflicht als einleitende Jobs des Argumentierens), (2) die der semantisch-pragmatischen Mittel, mit denen die Jobs von den einzelnen Interagierenden bewerkstelligt werden (z. B. Frage nach Gründen als Mittel für Etablieren von Begründungspflicht), (3) die der sprachlichen und körperlichen Formen, auf die die einzelnen Beteiligten in ihren Äußerungen konkret zurückgreifen (z. B. starkes Stirnrunzeln oder Warum-Frage als Ausdruck für das Einklagen von Begründungen). Mit Hilfe dieser Dreiteilung kann auch vergleichend auseinanderdividiert werden, welche Anteile die einzelnen Beteiligten jeweils an der Hervorbringung bestimmter Diskurseinheiten - z. B. Erklärungen oder Argumentationen - in Gesprächen übernehmen.

Die sich daraus ergebende Trennung zwischen ,gemeinsam hervorgebrachten Jobs und ,Eigenleistungen' des Kindes im Bereich seiner Mittel und Formen muss im Rahmen jeder interaktionsbasierten und mit Gesprächsdaten arbeitenden Erwerbstheorie analytisch beachtet werden. Die Modellierung der interaktionsbasierten Erwerbsmechanismen einerseits und der internen Ressourcen des Kindes andererseits führt zudem dazu, dass der Ressourcenbegriff unter ontogenetischen Aspekten differenziert werden kann: Wir unterscheiden zwischen externen und internen Ressourcen, die erst im Zusammenspiel a) zu einem geglückten Vollzug der Diskurseinheit eines bestimmten Typs bzw. einer bestimmten Gattung führen und b) über die Zeit zu Auf- und Ausbau diskursiver Kompetenzen auf Seiten 
des Kindes führen. Die erwähnten Partizipationsgelegenheiten (s. Abschnitt 3) werden mit diesem Zusammenspiel beschreibbar.

Die internen Ressourcen verweisen auf die bereits vorhandenen kognitiven und sprachlich-diskursiven Kompetenzen des Kindes, auf die es bei der Bearbeitung bestimmter diskursiver Anforderungen zurückgreift, sodass die entsprechenden selbstständigen Anteile des Kindes an der gemeinsamen diskursiven Aktivität möglich werden.

Externe Ressourcen des Diskurserwerbs hingegen sind zunächst die jeweils kontextuell zu nutzenden dialogischen Unterstützungen des Zuhörers oder der Zuhörerin und langfristig über die Ontogenese diejenigen habitualisierten interaktiven und diskursiven Strukturen konkreter individueller Lebenswelten, die die kindseitige Partizipation an bestimmten Diskurspraktiken eröffnen und steuern. Dazu zählen zum einen die jeweils für eine Interaktionsgemeinschaft rekurrent aktualisierten Gattungsrepertoires (s. Abschnitt 2), die überhaupt erst das Potenzial zur Partizipation an bestimmten Praktiken des Erklärens, Argumentierens usw. konstituieren: Wo nicht erklärt oder argumentiert wird, hat das Kind keine Chance, sich am Vollzug der entsprechenden Praktiken zu beteiligen. Weiterhin verstehen wir unter externen Erwerbsressourcen die tagtäglich erlebten erwerbsförderlichen Partizipationsstrukturen bzw. Interaktionsmuster, die beim Vollzug entsprechender diskursiver Gattungen sichtbar werden. Empirisch lassen sich unterschiedliche solcher Muster der Erwachsenen-Kind-Interaktion rekonstruieren (z. B. Fordern und Unterstützen, vgl. Quasthoff \& Kern 2007; Morek 2012; Übergehen und Selberlösen, vgl. Heller \& Krah 2015; Raumlassen und Akzeptieren, vgl. Quasthoff \& Kluger in diesem Band). Sie unterscheiden sich u. a. darin, welche Anteile an der Bearbeitung der für eine Gattung typischen gesprächsstrukturellen Jobs ein Kind nach Umfang und Art jeweils typischerweise übernimmt und welche von anderen Interaktionspartnern, z. B. Eltern oder Lehrpersonen, beigesteuert werden. Daran bemisst sich u. a. das Ausmaß der Erwerbsunterstützung für das in unterschiedlichem Maß partizipierende Kind.

Gesprächsbeiträge der Erwachsenen gewinnen in dieser Betrachtungsweise den Charakter von kontextuellen Steuerungen, die kindliche Gesprächsbeiträge sequenziell fordern, nahelegen oder auch verhindern - kurz: Partizipationsgelegenheiten schaffen oder verschließen und damit als externe Ressource dienen. Als erwerbsförderlich erweisen sich ,fordernd-unterstützende‘ Gesprächsaktivitäten des Erwachsenen (vgl. Quasthoff \& Kluger in diesem Band). Sie zeichnen sich durch den kontingenten Zuschnitt auf die kommunikativen Beiträge des Kindes aus: Der/die Erwachsene fragt erst dann nach oder formuliert selbst etwa eine Vermutung, wenn das Kind seinerseits die aus seiner Beteiligungsrolle erwartbaren Züge im Gespräch nicht selbst anschließt. Damit gewährleistet 
das Muster Fordern und Unterstützen - ontogenetisch betrachtet - die Anpassung an den Erwerbsstand des Kindes.

Wir haben diese Arbeitsteilung zwischen Kind und Erwachsenem bei der Erledigung der konversationellen Arbeit, bei der sich mit zunehmender Kompetenz der Kinder die Anteile immer mehr angleichen, in der Metapher der Wippe gefasst (Hausendorf \& Quasthoff 1996; Quasthoff 2011). Erst ein solches mikrostrukturell-adaptives Agieren im Sinne eines Finetunings (Bruner 1983; Morek \& Heller in diesem Band) entfaltet erwerbsunterstützendes Potenzial.

Insgesamt dienen die externen Ressourcen in Form der steuernden dialogischen Unterstützungen seitens der oder des Erwachsenen einerseits der Herstellung bzw. Sicherung von Verständigung: Der/die ,schwerere‘ (d. h. kompetentere) Erwachsene muss umso mehr, wippen', je leichter (d. h. weniger kompetent) das Kind ist, damit die kommunikativen Zwecke in der wechselseitigen Interaktion überhaupt umgesetzt werden können. Andererseits bieten genau diese auf das Kind austarierten dialogischen Unterstützungen für den kommunikativen Erfolg dem Kind Diskurserwerbsgelegenheiten - dies allerdings, wie entsprechende Vergleichsstudien zeigen, in stark variierendem Maße (vgl. Morek in diesem Band).

\section{Heterogenität außerschulischer Diskurserfahrungen und Partizipation im Unterricht}

Empirische Studien zu Diskurssozialisation und Diskurserwerb dokumentieren: Art und Ausmaß der Partizipation von Kindern und Jugendlichen an diskursiven Praktiken (vgl. Abschnitt 2) variieren ebenso, wie es auch Erwerbsverläufe und Kompetenzstände im Bereich mündlicher und schriftlicher Diskursfähigkeiten tun (vgl. Quasthoff \& Kern 2007; Heller 2012; Morek 2012; Quasthoff et al. 2019). Für den vorliegenden Band stehen vor allem interindividuelle Unterschiede im Bereich diskursiver Partizipation und Partizipationsgelegenheiten im Fokus (zu Varianten in der Kompetenzentwicklung vgl. Quasthoff et al. 2019).

Gezielte Einblicke in die Heterogenität außerschulischer sprachlich-diskursiver Erwerbsressourcen erlangen wir mit der Untersuchung von Interaktionen in Familien (vgl. Heller 2012; Quasthoff, Wild et al. in diesem Band; Domenech \& Krah in diesem Band; Morek 2012, in diesem Band) und Peergroups (vgl. Morek 2014, in diesem Band) von Schülerinnen und Schülern. Mit dem Ziel, verfügbare externe Ressourcen zu ermitteln, lässt sich hier zum 
einen nach den jeweils beobachtbaren Gattungsrepertoires und der zu ihrem Vollzug notwendigen Vertextungs- und Markierungsmuster fragen. Zum anderen interessieren die im Zuge diskursiver Praktiken vollzogenen mehr oder weniger erwerbsunterstützenden Interaktionsmuster. Im ersten Fall wird untersucht, welche diskursiven Gattungen es sind, die in den lebensweltlichen und unterrichtlichen Kontexten verschiedener Kinder und Jugendlicher überhaupt praktiziert werden. Hier zeigt sich: Nur für einige Kinder gehören beispielsweise Argumentieren und Erklären auch zum außerunterrichtlichen Erfahrungsraum - und nicht immer werden diese Gattungen, wenn sie denn vorkommen, mit sprachlichen Mitteln und entlang von Vertextungsverfahren (Heller in diesem Band) vollzogen, die unterrichtlichen Anforderungen an Explizitheit und Vollständigkeit entsprechen.

Zweitens lassen sich mit GLOBE Varianzen in Interaktionsmustern und damit Beteiligungsstrukturen beim Vollzug der jeweiligen Diskursaktivitäten rekonstruieren. Als unterschiedlich erwerbsförderliche Muster zeigten sich in unterschiedlichen Korpora u. a. Fordern und Unterstützen sowie Übergehen und Selberlösen sowie Varianten davon, die jeweils andere Partizipationsspielräume und -anforderungen für die kindlichen Gesprächspartner enthalten (Quasthoff \& Kern 2007; Quasthoff \& Kluger in diesem Band). Solche mikroanalytisch herausgearbeiteten Muster lassen sich in Beziehung setzen zu Befunden zur kindlichen Kompetenzentwicklung (Quasthoff \& Wild 2014; Domenech \& Krah in diesem Band; Quasthoff, Wild et al. in diesem Band; Quasthoff \& Kluger in diesem Band).

Wie sind nun die heterogenen außerschulischen Diskurserfahrungen und Partizipationsmöglichkeiten von Kindern und Jugendlichen unter dem Passungsgesichtspunkt - Unterricht als gatekeeper für Bildungsteilhabe - zu betrachten? Den älteren ethnographisch und anthropologisch orientierten Arbeiten zu Passungen und Divergenzen zwischen außerschulischen und schulischen Sprach- und Diskurserfahrungen folgend kann hier zunächst der oben bereits angesprochene Abgleich zwischen unterrichtlichen Anforderungen und ,mitgebrachten' außerschulischen Diskurs- und Partizipationsmustern in den Fokus gerückt werden. In dieser eher statischen Betrachtung können die Gattungsrepertoires, denen Kinder im Unterricht sowie in Familie und Peergroup begegnen, in unterschiedlichem Maße kongruent bzw. kompatibel sein (vgl. auch Heath 1983; Lareau 2003). Es lassen sich aber auch unterschiedliche Grade der Passung zwischen kontextuell typischerweise erwarteten Partizipationsstrukturen aufdecken. Eine Pionierarbeit stellt in diesem Zusammenhang die Untersuchung von Philips (1972) dar, die markante Unterschiede zwischen den Partizipationsstrukturen im US-amerikanischen Unterricht und denen in der community indianischer Kinder in Warm Springs offenlegt. Die Partizipati- 
onsstrukturen im Unterricht sehen vor allem vor, dass jeweils nur Einzelne sprechen - die Lehrperson bei Instruktionen und Lehrervorträgen oder die Lernenden beim Erklären bzw. der Demonstration von Wissen. Demgegenüber sind in der community Aktivitäten zumeist kooperativ organisiert und sehen auch für Kinder vielfältige Gelegenheiten der aktiven Partizipation vor. Philips sieht in den divergierenden Partizipationsstrukturen einen Erklärungsansatz für die schwachen Schulleistungen der indianischen Kinder. Mit kulturell unterschiedlichen Erzählpraktiken im Vergleich zu den Erwartungen der Lehrpersonen erklären Collins \& Michaels (1984) in ähnlicher Weise Benachteiligungen afro-amerikanischer Kinder.

Ein derart statisches Verständnis kann jedoch den dynamischen Prozessen der situativen Aushandlung und Ko-Konstruktion von Passung zwischen schülerseitigen Ressourcen und unterrichtlichen Erwartungen in situ nicht hinreichend Rechnung tragen. Dies belegen v. a. ethnomethodologisch und konversationsanalytisch orientierte Studien (z. B. Heller 2012; Buttlar 2017, Leßmann in diesem Band; Heller \& Kotthoff 2020). In einem dynamisierten Verständnis von Passung richtet sich der Blick hier auf die wechselseitige Schaffung von Partizipationsgelegenheiten im Rahmen diskursiver Praktiken des Unterrichtsgesprächs sowie auf die lehrerseitige Herstellung von Passung durch Bearbeitung der Schülerbeiträge. Letztere werden sehr wesentlich (mit)bestimmt durch kommunikative ,Investitionen“ (Heller 2012: 55), die einzelnen Lernenden lehrerseitig entgegengebracht oder vorenthalten werden (z. B. Nachfragen, Reparaturen). In diesem Zusammenhang eines dynamischen Passungsbegriffs zeigt Heller (2012), dass gerade Kinder, die in ihrer familialen Interaktion aus unterschiedlichen Gründen nur wenige Ressourcen für die Partizipation an Unterrichtsgesprächen erworben haben, nicht nur von sich aus wenig partizipieren, sondern auch von der Lehrperson kaum aktiv involviert werden. Lehrpersonen fungieren somit gleichsam als ,Passungsagenten', die Angemessenheit von Schülerbeiträgen im Unterrichtsgespräch und damit Erwerbskontexte für sprachlich-diskursives Lernen sehr wesentlich etablieren oder beschränken. Damit ist eine Verbindung zwischen statischen und dynamischen, makrostrukturellen und mikroprozessualen Passungsverhältnissen empirisch in den Blick gerückt.

Solche unterrichtlichen Mechanismen der interaktiven Herstellung von Passung und Diskurspartizipation können in ihrer Dynamik auch über längere Zeiträume verfolgt und in ihren Auswirkungen auf unterschiedliche Lernchancen beschrieben werden: Zeichnet man längsschnittlich trajectories of participation (Cekaite 2012) nach, zeigt sich, dass die unterrichtliche Partizipation von Kindern, deren diskursive Erfahrungen nicht zu den schulischen Erwartungen und Anforderungen passen, über die Zeit zurückgeht, wenn Lehrpersonen nicht proaktiv Partizipationsgelegenheiten schaffen und interaktive Unterstützung - auch 
in Form von expliziten Rückmeldungen zu erwartungsdiskrepanten Beiträgen gewähren (Heller 2017; Quasthoff \& Prediger 2017). Typischerweise beschränken sich die Partizipationsgelegenheiten vor allem auf diejenigen Kinder, die sich ihrerseits aktiv mit passenden Beiträgen beteiligen und sich auch durch Fehlschläge nicht beirren lassen, also auch ihrerseits viel investieren (Heller 2017; Leßmann demn.). Darüber hinaus konnte gezeigt werden, dass diese Kinder über eine vergleichsweise hohe transsituative Kontextualisierungskompetenz, d.h. die Fähigkeit zum situativ angemessenen sprachlichen Handeln in unterschiedlichen sozialen Kontexten, verfügen (Quasthoff \& Morek 2015). Derartige Befunde sind von hoher Relevanz für Überlegungen zur Ableitung von Unterstützung und Fördermaßnahmen im Bereich diskursiver Unterstützung benachteiligter Schülerinnen und Schüler.

\section{Ausblick: Von der Rekonstruktion zur Vermittlung}

Die diesem Band primär zugrundeliegenden Studien (vgl. Einführung in den Band) sind allesamt mit einem deskriptiv-explanativen Anliegen in der Grundlagenforschung gestartet. Ihr Ziel war in unterschiedlicher Fokussierung die Aufklärung des Zusammenhangs von diskursiven Kompetenzen mit der Partizipation an bildungsrelevanten Diskurspraktiken und damit letztlich der Teilhabe am schulisch vermittelten Bildungserfolg. Die Untersuchung real ablaufender sprachlicher Interaktionen in Familien-, Peer- und Unterrichtsinteraktionen eröffnet dabei nicht nur Einsichten in Art und Ausmaß heterogener Diskurs- und Interaktionserfahrungen, sondern fördert vor allem auch zutage, welche Formen diskursiven Partizipierens und Involvierens besonderes Erwerbs- bzw. Förderpotenzial mit sich bringen. Daraus ergeben sich unmittelbare Anwendungsperspektiven bezüglich der Frage, wie Kinder und Jugendliche beim Erwerb diskursiver Kompetenzen besonders gut unterstützt werden können.

Vor dem Hintergrund von Bildungsbe(nach)teiligung rückt dabei nicht nur, aber vor allem die Situation derjenigen Schülerinnen und Schüler ins Zentrum, die nachweislich schulisch wie außerschulisch nur eingeschränkte Partizipationschancen bei schulrelevanten diskursiven Praktiken wie Berichten, Erklären und Argumentieren erhalten. Hier ergeben sich im Wesentlichen zwei Ansatzpunkte, um auf die damit verbundenen reduzierten diskursiven Erwerbsgelegenheiten und damit die weiteren Partizipationschancen an Lehr-/Lerndiskursen zu reagieren: Einerseits kann man an außerschulischen Erwerbskontexten ansetzen und versuchen, habitualisierte familiale Interaktionsweisen zugunsten koopera- 
tions- und diskursförderlicherer Muster aufzubrechen (vgl. Kluger in diesem Band). Andererseits kann man das Augenmerk verstärkt auf Unterricht - und hier speziell auf mündliche Interaktionsprozesse - als Lernort für fachliche und diskursive Kompetenzen legen (vgl. Morek \& Heller in diesem Band, i. Dr.). In beiden Fällen ist das Ziel die Vermittlung diskurserwerbsförderlicher Gesprächsführungsstrategien an die erwachsenen Interaktanten (vgl. Kluger in diesem Band; Prediger in diesem Band).

Für Eltern-Kind-Interaktionen liegt bereits ein ausgebautes Trainingsprogramm vor, das familiales Argumentieren fokussiert und dabei systematisch auf die Erhöhung kindlicher diskursiver und sozialer Partizipation gerichtet ist (vgl. Kluger in diesem Band). Mikroanalytische Untersuchungen von Fachunterrichtssequenzen zeigen zudem, dass auch manche Lehrkräfte - wie etwa $50 \%$ der Eltern - durchaus auf kontextualisierte Elemente des Musters Fordern und Unterstützen zurückgreifen (Heller \& Morek 2015b) und ihre Gesprächsaktivitäten und Zuhörerreaktionen auf Erwerbsstände unterschiedlichster Schülerinnen und Schüler adaptieren (vgl. Morek \& Heller in diesem Band). Auf diese für professionelle Qualifizierungen nutzbare Weise können im Unterrichtsgespräch Partizipationsgelegenheiten für alle Schülerinnen und Schüler geschaffen werden.

Es gibt also ermutigende Hinweise darauf, dass - und wie - es gelingen kann, diskursive Passung und Partizipation von Kindern und Jugendlichen mit und in ihren unterschiedlichen Lebenswelten zu erhöhen. Die in diesem Band zusammengefasste Integration von methodologischer und erwerbsorientierter Grundlagenforschung mit nachfolgend entwickelten und empirisch teilweise bereits als wirksam nachgewiesenen Fördermaßnahmen mögen hierzu einen Beitrag leisten.

\section{Literatur}

Bergmann, Jörg (1987): Klatsch. Zur Sozialform der diskreten Indiskretion. Berlin: de Gruyter. Bernstein, Basil (1977): Class, codes and control. London: Routledge.

Blum-Kulka, Shoshana (1997): Dinner talk. Patterns of sociability and socialization in family discourse. Mahwah, NJ: Erlbaum.

Blum-Kulka, Shoshana \& Catherine Snow (Hrsg.) (2002): Talking to Adults. The Contribution of Multiparty Discourse to Language Acquisition. Mahwah, NJ: Erlbaum.

Bourdieu, Pierre (1977): Cultural Reproduction and Social Reproduction. In Jerome Karabel \& Albert Halsey (Hrsg.), Power and ideology in education, 487-510. New York: Oxford University Press.

Bourdieu, Pierre \& Jean-Claude Passeron (1977): Reproduction in education, society and culture. London: Sage.

Bruner, Jerome (1983): Child's talk. Learning to use language. New York: Norton. 
Burdelski, Matthew \& Kathryn Howard (Hrsg.) (2020): Language socialization in classrooms. Culture, interaction, and language development. Cambridge: Cambridge University Press.

Buttlar, Ann-Christin (2017): Implizite Normvermittlung durch Herstellung von Angemessenheit im Unterrichtsdiskurs. In Stefan Hauser \& Martin Luginbühl (Hrsg.), Gesprächskompetenz in schulischer Interaktion - normative Ansprüche und kommunikative Praktiken, 38-64. Bern: hep.

Cekaite, Asta (2012): Affective Stances in Teacher-Novice Student Interactions. Language, Embodiment, and Willingness to Learn in a Swedish Primary Classroom. Language in Society 41 (5), 641-670.

Collins, James \& Sarah Michaels (1984): Oral discourse styles: Classroom interaction and acquisition of literacy. In Deborah Tannen (Hrsg.), Coherence in spoken and written discourse, 219-244. Norwood, NJ: Ablex.

Feilke, Helmuth (2012): Bildungssprachliche Kompetenzen - fördern und entwickeln. Praxis Deutsch 233, 4-13.

Garfinkel, Harold (1967): Studies in ethnomethodology. Cambridge: Polity Press.

Goffman, Erving (1981): “Footing”. In Erving Goffman (Hrsg.), Forms of talk, 124-159. Philadelphia: University of Pennsylvania Press.

Gogolin, Ingrid (2010): Was ist Bildungssprache? Grundschulunterricht Deutsch 57 (4), 4-5

Goldberg, Jo (1975): A system for the transfer of instructions in natural settings. Semiotica 14 (3), 269-296.

Goodwin, Charles (2007): Interactive footing. In Elizabeth Holt \& Rebecca Clift (Hrsg.), Reporting talk. Reported speech in interaction, 16-46. Cambridge: Cambridge University Press.

Goodwin, Marjorie \& Asta Cekaite (2018): Embodied family choreography. Practices of control, care, and mundane creativity. London, New York: Routledge.

Goodwin, Charles \& Marjorie Goodwin (2004): Participation. In Alessandro Duranti (Hrsg.), A companion to linguistic anthropology, 222-244. Malden, Mass.: Blackwell.

Goodwin, Marjorie \& Charles Goodwin (1987): Children's Arguing. In Susan Philips, Susan Steele \& Christine Tanz (Hrsg.), Language, gender, and sex in comparative perspective, 200-248. Cambridge: Cambridge University Press.

Grøver, Vibeke, Paola Uccelli, Meredith Rowe \& Elena Lieven (Hrsg.) (2019): Learning through language. Towards an educationally informed theory of language learning. Cambridge: Cambridge University Press.

Günthner, Susanne (1995): Gattungen in der sozialen Praxis. Die Analyse „kommunikativer Gattungen“ als Textsorten mündlicher Kommunikation. Deutsche Sprache (3), 193-218.

Günthner, Susanne (2005): Narrative reconstructions of past experiences: Adjustments and modifications in the process of recontextualizing a past experience. In Uta Quasthoff \& Tabea Becker (Hrsg.), Narrative interaction, 285-301. Amsterdam: Benjamins.

Günthner, Susanne (2009): Intercultural communication and the relevance of cultural specific repertoires of communicative genres. In Helga Kotthoff \& Helen Spencer-Oatey (Hrsg.), Handbook of intercultural communication, 127-152. Berlin: De Gruyter.

Günthner, Susanne \& Hubert Knoblauch (1996): Die Analyse kommunikativer Gattungen in Alltagsinteraktionen. In Susanne Michaelis \& Doris Tophinke (Hrsg.), Texte Konstitution, Verarbeitung, Typik, 35-57. München: Lincom. 
Harren, Inga (2015): Fachliche Inhalte sprachlich ausdrücken lernen. Sprachliche Hürden und interaktive Vermittlungsverfahren im naturwissenschaftlichen Unterrichtsgespräch in der Mittel- und Oberstufe. Radolfzell: Verlag für Gesprächsforschung (Verfügbar unter: verlag-gespraechsforschung.de/2015/harren.html).

Hart, Betty \& Todd Risley (1999): The Social World of Children: Learning To Talk. Baltimore, MD: Paul H. Brookings Publishing.

Hausendorf, Heiko \& Uta Quasthoff (1996): Sprachentwicklung und Interaktion. Eine linguistische Studie zum Erwerb von Diskursfähigkeiten. Opladen: Westdeutscher Verlag. Neu aufgelegt: http://www.verlag-gespraechsforschung.de/2005/quasthoff.htm. Radolfzell: Verlag für Gesprächsforschung.

Heath, Shirley (1983): Ways with words. Language, life, and work in communities and classrooms. Cambridge: Cambridge University Press.

Heller, Vivien (2012): Kommunikative Erfahrungen von Kindern in Familie und Unterricht. Passungen und Divergenzen. Tübingen: Stauffenburg.

Heller, Vivien (2017): Lerngelegenheiten für bildungssprachliche Kompetenzen: Wie partizipieren DaZ-Lerner am Erklären und Argumentieren im Unterricht? In Isabel Fuchs, Stefan Jeuk \& Werner Knapp (Hrsg.), Mehrsprachigkeit: Spracherwerb, Unterrichtsprozesse, Schulentwicklung, 165-182. Stuttgart: Fillibach.

Heller, Vivien \& Helga Kotthoff (2020): Ethnografien und Interaktionsanalysen im schulischen Feld. Zur Einführung in den Band. In Helga Kotthoff \& Vivien Heller (Hrsg.), Ethnografien und Interaktionsanalysen im schulischen Feld. Diskursive Praktiken und Passungen interdisziplinär, 7-24. Tübingen: Narr.

Heller, Vivien \& Antje Krah (2015): Wie Eltern und Kinder argumentieren. Interaktionsmuster und ihr erwerbssupportives Potenzial im längsschnittlichen Vergleich. Mitteilungen des Deutschen Germanistenverbandes 62 (1), 5-20.

Heller, Vivien \& Miriam Morek (2015a): Academic discourse as situated practice: An introduction. Linguistics and Education 31, 174-186.

Heller, Vivien \& Miriam Morek (2015b): Unterrichtsgespräche als Erwerbskontext: Kommunikative Gelegenheiten für bildungssprachliche Praktiken erkennen und nutzen. leseforum.ch (3), 1-23.

Heller, Vivien, Uta Quasthoff, Susanne Prediger \& Anna Vogler (2017): Bildungssprachliche Praktiken aus professioneller Sicht: Wie deuten Lehrende Erklärungen und Begründungen von Kindern? In Bernt Ahrenholz, Britta Hövelbrinks \& Claudia Schmellentin (Hrsg.), Fachunterricht und Sprache in schulischen Lehr-/Lernprozessen, 139-160. Tübingen: Narr.

Hoff-Ginsberg, Erika (1991): Mother-child conversation in different social classes and communicative settings. Child development 62 (4), 782-796.

Keppler, Angela \& Thomas Luckmann (1991): Teaching: Conversational transmission of knowledge. In Ivana Markovà \& Klaus Foppa (Hrsg.), Asymmetries in dialogue, 143-166. Savage: Barnes \& Noble.

Kleinschmidt-Schinke, Katrin (2018): Die an die Schüler/-innen gerichtete Sprache (SgS). Studien zur Veränderung der Lehrer/-innensprache von der Grundschule bis zur Oberstufe. Berlin: De Gruyter.

Klette, Kirsti, Fritjof Sahlström, Marte Blikstad-Balas, Jennifer Luoto, Marie Tanner \& Michael Tengberg (2018): Justice through participation. Student engagement in Nordic classrooms. Education Inquiry 9 (1), 57-77. 
Knoblauch, Hubert (1991): The taming of the foes: The avoidance of asymmetry in informal discussions. In Ivana Markovà \& Klaus Foppa (Hrsg.), Asymmetries in dialogue, 166-194. Savage: Barnes \& Noble.

Kotthoff, Helga (2009): Gesprächsfähigkeiten: Erzählen, Argumentieren, Erklären. In Michael Krelle \& Carmen Spiegel (Hrsg.), Sprechen und Kommunizieren. Entwicklungsperspektiven, Diagnosemöglichkeiten und Lernszenarien in Deutschunterricht und Deutschdidaktik, 41-63. Baltmannsweiler: Schneider Hohengehren.

Kramer, Rolf \& Werner Helsper (2010): Kulturelle Passung und Bildungsungleichheit Potenziale einer an Bourdieu orientierten Analyse der Bildungsungleichheit. In HeinzHermann Krüger, Ursula Rabe-Kleberg, Rolf Kramer \& Jürgen Budde (Hrsg.), Bildungsungleichheit revisited. Bildung und soziale Ungleichheit vom Kindergarten bis zur Hochschule, 103-125. Wiesbaden: VS.

Lareau, Annette (2003): Unequal childhoods. Class, race, and family life. Berkeley: University of California Press.

Leßmann, Ann-Christin (demn.): Unterrichtsinteraktion in der Grundschule. Sequenzielle Analysen zur Ko-Konstruktion von Angemessenheit zwischen Lehrenden und Lernenden. Tübingen: Stauffenburg.

Levinson, Steven (1988). Putting linguistics on a proper footing: Explorations in Goffman's concepts of participation. In Paul Drew \& Anthony Wootton (Hrsg.), Erving Goffman. Exploring the interaction order, 161-227. Cambridge: Polity Press.

Luckmann, Thomas (1986): Grundformen der gesellschaftlichen Vermittlung des Wissens: Kommunikative Gattungen. Kölner Zeitschrift für Soziologie und Sozialpsychologie 27, 191-211.

Luckmann, Thomas (1989): Kultur und Kommunikation. In Max Haller, Wolfgang Zapf \& HansJoachim Hoffmann-Nowotny (Hrsg.): Kultur und Gesellschaft, 33-45. Frankfurt: Campus.

Morek, Miriam (2012): Kinder erklären. Interaktionen in Familie und Unterricht im Vergleich. Tübingen: Stauffenburg.

Morek, Miriam (2014): Constructing social and communicative worlds - The role of peerinteractions in preadolescents' discursive development. Learning, Culture and Social Interaction 3 (2), 121-133.

Morek, Miriam (2016): Formen mündlicher Darstellung in situ: Zur Komplexität von Diskursanforderungen in Unterrichtsgesprächen. In Ulrike Behrens \& Olaf Gätje (Hrsg.), Mündliches und schriftliches Handeln im Deutschunterricht. Wie Themen entfaltet werden. 95-131. Frankfurt a.M.: Lang.

Morek, Miriam (2018): Multimodalität als Ressource der Platzierung von Erzählungen in PeerInteraktionen von Kindern. Gesprächsforschung - Online-Zeitschrift zur verbalen Interaktion 19, 304-345.

Morek, Miriam \& Vivien Heller (2012): Bildungssprache - Kommunikative, epistemische, soziale und interaktive Aspekte ihres Gebrauchs. Zeitschrift für angewandte Linguistik 57 (1), 67-101.

Morek, Miriam \& Vivien Heller (2019): Bildungssprachliche Praktiken. In Anja Ballis, Johanna Meixner \& Meike Heithorst (Hrsg), Glossar "Sprache im Fach", 1-3 (Online verfügbar unter https://spracheimfach.de/glossar/, letzter Zugriff: 18. 03.2020)

Morek, Miriam \& Vivien Heller (i. Dr.): Überlegungen zur Professionalisierung für unterrichtliche Gesprächsführung - (Wie) Können Lehrkräfte diskurserwerbsförderliches Lehrerhandeln lernen? In Frederike Schmidt \& Kirsten Schindler (Hrsg.), Wissen und 
Überzeugungen von Deutschlehrkräften. Aktuelle Befunde in der deutschdidaktischen Professionsforschung, 229-245. Frankfurt a.M.: Lang.

Morek, Miriam, Vivien Heller \& Uta Quasthoff (2017): Erklären und Argumentieren Modellierungen und empirische Befunde im Rahmen der linguistischen Erwerbs- und Unterrichtsforschung. In Iris Meißner \& Eva Wyss (Hrsg.), Begründen - Erklären Argumentieren. Konzepte und Modellierungen in der Angewandten Linguistik, 11-46. Tübingen: Stauffenburg.

Pauli, Christine \& Kurt Reusser (2015): Discursive Cultures of Learning in (Everyday) Mathematics Teaching: A Video-Based Study on Mathematics Teaching in German and Swiss Classrooms. In Lauren Resnick, Christa Asterhan \& Sherice Clarke (Hrsg.), Socializing intelligence through academic talk and dialogue, 181-193. Washington, DC: American Educational Research Association.

Philips, Susan (1972): Participant structures and communicative competence: Warm Springs children in community and classroom. In Courtney Cazden, Vera John \& Dell Hymes (Hrsg.), Functions of language in the classroom, 86-127. Prospect Heights: Waveland Press.

Pontecorvo, Clotilde \& Alessandra Fasulo (1997): Learning to Argue in Family Shared Discourse: The Reconstruction of Past Events. In Lauren Resnick (Hrsg.), Discourse, tools, and reasoning: essays on situated cognition, 406-442. Berlin: Springer.

Psathas, George (1992): The Study of Extended Sequences: The Case of the Garden Lesson. In Graham Watson \& Robert Seiler (Hrsg.), Text in context. Contributions to ethnomethodology, 99-122. Newbury Park: Sage.

Quasthoff, Uta (1980): Erzählen in Gesprächen. Linguistische Untersuchungen zu Strukturen und Funktionen am Beispiel einer Kommunikationsform des Alltags. Tübingen: Narr.

Quasthoff, Uta (1981): Zuhöreraktivitäten beim konversationellen Erzählen. In Peter Schröder \& Hugo Steger (Hrsg.), Dialogforschung, 287-313. Düsseldorf: Schwann.

Quasthoff, Uta (2011): Diskurs- und Textfähigkeiten: Kulturelle Ressourcen ihres Erwerbs. In Ludger Hoffmann, Kerstin Leimbrink \& Uta Quasthoff (Hrsg.), Die Matrix der menschlichen Entwicklung, 210-251. Berlin: De Gruyter.

Quasthoff, Uta, Vivien Heller \& Miriam Morek (2017): On the sequential organization and genre-orientation of discourse units in interaction: An analytic framework. Discourse Studies 19 (1), 84-110.

Quasthoff, Uta \& Friederike Kern (2007): Familiale Interaktionsmuster und kindliche Diskursfähigkeit: Mögliche Auswirkungen interaktiver Stile auf diskursive Praktiken und Kompetenzen bei Schulkindern. In Heiko Hausendorf (Hrsg.), Gespräch als Prozess. Linguistische Aspekte der Zeitlichkeit verbaler Interaktion, 277-306. Tübingen: Narr.

Quasthoff, Uta, Friederike Kern, Sören Ohlhus \& Juliane Stude (2019): Diskurse und Texte von Kindern: Praktiken - Fähigkeiten - Ressourcen: Erwerb. Tübingen: Stauffenburg Verlag. http://dx.doi.org/10.17877/DE290R-20360.

Quasthoff, Uta \& Miriam Morek (2015): Diskursive Praktiken von Kindern in außerschulischen und schulischen Kontexten. Abschlussbericht. TU Dortmund. https://www.disko.tu-dort mund.de/disko/Medienpool/Abschlussbericht-DisKo.pdf

Quasthoff, Uta \& Susanne Prediger (2017): Fachbezogene Unterrichtsdiskurse zu Beginn der weiterführenden Schule - Interdisziplinäre Untersuchungen zur Unterstützung von sprachlichem und fachlichem Lernen. In Winfried Thielmann, Caroline Trautmann, Arne 
Krause \& Gesa Lehmann (Hrsg.), Form und Funktion. Festschrift für Angelika Redder, 625-644. Tübingen: Stauffenburg.

Quasthoff, Uta \& Elke Wild (2014): Learning in context from an interdisciplinary perspective. Learning, Culture and Social Interaction 3, 69-76.

Schleppegrell, Mary (2012): Academic Language in Teaching and Learning. The Elementary School Journal 112 (3), 409-418.

Sacks, Harvey (1995): Lectures on conversation. Oxford, UK, Cambridge: Blackwell.

Schütz, Alfred \& Thomas Luckmann (1975): Strukturen der Lebenswelt. Neuwied: Luchterhand. Spranz-Fogasy, Thomas (2003): Alles Argumentieren, oder was? Zur Konstitution von Argumentation in Gesprächen. In Arnulf Deppermann \& Martin Hartung (Hrsg.), Argumentieren in Gesprächen. Gesprächsanalytische Studien, 27-39. Tübingen: Stauffenburg.

Stanat, Petra, Katrin Böhme, Stefan Schipolowski \& Nicole Haag (Hrsg.) (2016): IQBBildungstrend 2015. Sprachliche Kompetenzen am Ende der 9. Jahrgangsstufe im zweiten Ländervergleich. Münster: Waxmann.

Stanat, Petra, Stefan Schipolowski, Camilla Rjosk, Sebastian Weirich \& Nicole Haag (Hrsg.) (2017): IQB-Bildungstrend 2016. Kompetenzen in den Fächern Deutsch und Mathematik am Ende der 4. Jahrgangsstufe im zweiten Ländervergleich. Münster: Waxmann.

Wald, Benji (1978): Zur Einheitlichkeit und Einleitung von Diskurseinheiten. In Uta Quasthoff (Hrsg.), Sprachstruktur - Sozialstruktur, 128-149. Königstein: Scriptor. 